\title{
Islet Amyloid Development in a Mouse Strain Lacking Endogenous Islet Amyloid Polypeptide (IAPP) but Expressing Human IAPP
}

\author{
Gunilla T. Westermark, ${ }^{1}$ Samuel Gebre-Medhin, ${ }^{2}$ Donald F. Steiner, ${ }^{4}$ \\ and Per Westermark ${ }^{3}$ \\ ${ }^{1}$ Division of Medical Cell Biology, Department of Biomedicine and Surgery, \\ Linköping University, Linköping, Sweden \\ ${ }^{2}$ Department of Physiological Sciences, Division of Molecular and Cellular \\ Physiology, Lund University \\ ${ }^{3}$ Department of Genetics and Pathology, Uppsala University, Uppsala, Sweden \\ ${ }^{4}$ Department of Biochemistry and Howard Hughes Medical Institute, University \\ of Chicago, Chicago, Illinois, U.S.A.
}

Accepted September 8, 2000.

\begin{abstract}
Background: Several mouse strains expressing human islet amyloid polypeptide (IAPP) have been created to study development of islet amyloid and its impact on islet cell function. The tendency to form islet amyloid has varied strongly among these strains by factors that have not been elucidated. Because some beta cell granule components are known to inhibit IAPP fibril formation in vitro, we wanted to determine whether a mouse strain expressing human IAPP but lacking the nonamyloidogenic mouse IAPP is more prone to develop islet amyloidosis.

Materials and Methods: Such a strain was created by cross-breeding a transgenic mouse strain and an IAPP null mouse strain.
\end{abstract}

\section{Introduction}

Islet amyloid polypeptide (IAPP, amylin) is a regulatory peptide, expressed by pancreatic islet beta cells and stored and released together with insulin (1). The normal function of IAPP is insufficiently understood but experimentally many different effects have been described

Address correspondence and reprint requests to: Gunilla T. Westermark, Division of Medical Cell Biology, University Hospital, S-581 85 Linköping, Sweden. Fax: +46-13-22 41 49; E-mail: gunwe@IBK.liu.se
Results: When fed a fat-enriched diet, male mice expressing only human IAPP developed islet amyloid earlier and to a higher extent than did mice expressing both human and mouse IAPP. Supporting these results, we found that mouse IAPP dose-dependently inhibits formation of fibrils from human IAPP.

Conclusions: Female mice did not develop amyloid deposits, although small extracellular amorphous IAPP deposits were found in some islets. When cultivated in vitro, amyloid deposits occurred within 10 days in islets from either male or female mice expressing only human IAPP. The study shows that formation of islet amyloid may be dependent on the environment, including the presence or absence of fibril inhibitors or promoters.

(e.g., on insulin and glucagon release, peripheral glucose utilization, satiety, and gastric emptying) (2).

IAPP was discovered as a component of extracellular amyloid deposits in the islets of Langerhans of diabetic individuals (3). This is a long recognized pathological phenomenon in which islets may gradually be converted to amyloid. Islet amyloid deposition is seen in conjunction with aging but is especially common and pronounced in association with type 2 diabetes mellitus. Up to $95 \%$ of individuals 
with type 2 diabetes have been found to have some degree of islet amyloidosis (4). Islet amyloid was long ignored as a possible pathogenetic factor in diabetogenesis. However, an increasing interest in this pathological feature has arisen in recent years, and islet amyloid is now generally considered to be of pathogenic importance for the late beta cell impairment in type 2 diabetes $(5,6)$.

The mechanism underlying the aggregation of normally soluble IAPP molecules into insoluble amyloid fibrils in vivo is unclear. The 37 amino acid residue IAPP has a central segment (residues 20-29) that determines its aggregation properties (7). Amino acid variations in this segment explain why IAPP only gives rise to islet amyloid in certain species. Specifically, proline residues at positions 24 and 28 as in the rat and mouse IAPP are believed to disrupt $\beta$-structure, a necessary feature for amyloid fibril formation, whereas the amino acid sequences found in human and cat IAPP facilitate amyloidogenesis (8).

No easily available animal models exist for the study of pathogenesis or impact of the islet amyloid. Because human IAPP (hIAPP) is amyloidogenic whereas mouse IAPP (mIAPP) is not, several transgenic mouse strains have been created to study the process of amyloidogenesis and the subsequent islet lesions (9-12). The constructs have varied but all expression of IAPP has been directed to the beta cells by the use of insulin gene promoters. Consequently, the production of IAPP in these mice has been much higher than normal resulting in a higher than normal IAPP to insulin ratio in the pancreas and plasma. However, the occurrence of amyloid deposition in these transgenic mouse strains has been highly variable. In one of the strains, islet amyloid has been reported to occur when the animals have been placed on a high fat diet (13). In other strains, amyloid developed when the animals were treated with growth hormone or when crossed into insulin-resistant mouse strains (14-16). In one of the strains, islet amyloid has been reported to occur spontaneously in male homozygous mice (15). Other strains, despite comparably high levels of hIAPP, fail to develop significant islet amyloid deposits.

We have been working with a transgenic mouse strain, which expresses hIAPP driven by the rat insulin I promoter (11). This mouse strain has plasma IAPP levels that are 5-10 times higher than normal. The mice, however, do not develop islet amyloid at any age, even if the animals have been made insulin-resistant by corticosteroid treatment or by crossing into ob/ob genetic background (17). Similar to another hIAPP-expressing mouse strain, islet amyloid forms rapidly when isolated islets from the mice are cultivated in vitro (17-19). The reasons for these discrepancies among the different transgenic mouse strains are unknown.

It is possible that the endogenous mIAPP may interfere with amyloid fibril formation. This is a not unreasonable possibility because we (20) as well as others (21) have carried out experiments that indicate that various beta cell granule components affect fibril formation from hIAPP in vitro. Recently, a mouse strain that lacks IAPP has been created by gene targeting (22). These mice have normal fasting blood glucose and plasma insulin concentrations and they reproduce normally. However, adult males of the IAPP null strain have a significantly increased plasma insulin response upon glucose loading (22). We have crossed this IAPP null mouse strain with homozygous hIAPP transgenic animals to create a mouse strain that expresses only hIAPP. In this communication, we provide some basic data regarding this novel strain, and demonstrate that these animals are more susceptible to islet amyloid formation than mice expressing both mIAPP and hIAPP.

\section{Materials and Methods}

Mouse Strain

A strain expressing hIAPP but not mIAPP was established by stepwise breeding of transgenic mice carrying the human IAPP gene (11) with IAPP null mutant (mIAPP-/-) mice (22). To assess the presence of the human IAPP gene, DNA was extracted from blood samples and analyzed by polymerase chain reaction (PCR) with the human IAPP-specific primers 5' AAATGCAACACTGCCACATGT and $3^{\prime}$ CCATATATTCACACTGGAG. Presence of the mouse IAPP gene was detected by Southern blotting (22). The mice used were inbred for at least ten generations, and were either homozygous $(+/+)$ or heterozygous $(+/-)$ with regard to the hIAPP gene.

\section{Weight Gain in Animals on Ordinary Diet}

The weight gain was monitored over 15 months in a group of 14 male hIAPP $+/-/$ mIAPP $-/-$ 
mice and in 14 male mIAPP-/- mice. These animals were housed individually with free access to standard chow and water and were kept on a 12-hr light and dark cycle.

\section{Effects of Fat-rich Diet}

Because islet amyloid was reported to occur in one hIAPP transgenic mouse strain after being fed a fat-rich diet (13), and because high-fat diet increases the plasma IAPP: insulin molar ratio in normal mice (23), animals were fed a fat-rich diet. The diet was based on lard and contained $24 \%$ fat (AnalyCen, Lidköping, Sweden). Control mice were fed normal standard chow containing $4.5 \%$ fat. The percentage of saturated fatty acids was $47 \%$ and $22 \%$, respectively. Thirty-eight hIAPP $+/+/$ mIAPP $-/-$ male mice were housed individually and divided into two equal groups each fed the separate diet. The weight gain and blood glucose levels were determined monthly. Six animals from each group were sacrificed after 6, 9, or 12 months (three animals died during the experimental time, and the group of five animals fed a low-fat diet were sacrificed after 12 months). Twenty-four hIAPP $+/+/$ mIAPP $-/$ - female mice were divided into two equal groups and fed different diets. The animals were housed in four groups, each containing six individuals. These animals were sacrificed after 9 and 12 months.

\section{Analyses}

Blood glucose concentrations were determined in blood samples obtained from the orbital sinus in mice that had been fasted for $12 \mathrm{hr}$. Animals were sacrificed by cervical dislocation after which the thoracic and abdominal cavities were opened and blood was collected from the heart. Pieces of the pancreas were fixed in formalin (vide infra). IAPP and insulin plasma concentrations were measured by radioimmunoassays as described (17).

\section{Pancreatic Tissue Analyses}

Pancreatic tissue was fixed in $10 \%$ buffered neutral formalin and embedded in paraffin. For the demonstration of amyloid in tissue sections, alkaline Congo red and thioflavin $\mathrm{T}$ were used on $10-\mu \mathrm{m}$ thick sections (24). Deparaffinized sections $(5 \mu \mathrm{m})$ were immunolabeled with guinea pig antiinsulin serum (Dako,
Copenhagen, Denmark; 1:1000). These sections were subsequently incubated with peroxidase conjugated rabbit anti-guinea pig antibodies (Dako) diluted 1:1000. Other sections were incubated with mouse monoclonal antibody to glucagon (Novaclone, Malmö, Sweden; 1:4000), rabbit anti-somatostatin serum(Dako; 1:2000) and anti-pancreatic polypeptide (PP) serum (Dako; 1:2000). Islet amyloid polypeptide immunoreactivity was visualized with the rabbit antisera A110 and A133, diluted 1:1000 and 1:400, respectively. Antiserum Al10 reacts with both human and mouse IAPP whereas Al33 is specific for human IAPP (17). The reactions were detected by incubation with biotinylated swine anti-rabbit antibodies or biotinylated goat anti-mouse antibodies 1:200 followed by streptavidinperoxidase (Dako; 1:500). 3,3'-diaminobenzidine tetrahydrochloride was used to visualize the reactions.

The islet areas and areas of cells containing insulin, glucagon, somatostatin, and pancreatic polypeptide were measured in pancreata from five male and five female hIAPP $+/+$ / mIAPP $-/-$ mice and from five male and five female IAPP deficient mice. In all mice, ten randomly chosen islets in each of three sections from different levels of the pancreatic tissue block were measured. For this, an image analysis program was applied as described (25). The areas of the respective cells were expressed as percentage of the islet area. The islet size was estimated by measuring the islet areas in sections stained with hematoxylin and eosin from three different levels of the pancreatic body.

\section{Amyloid Formation in Islets Cultivated In Vitro}

Islet amyloid has been mainly described to occur in male mice and to a much lesser extent in female mice. We wanted to investigate whether this also was true for amyloid developed in vitro.

Islets were isolated from six female and six male hIAPP $+/+/$ mIAPP $-/-$ mice, 3-6 months of age. Pancreatic tissue was excised and cut into small pieces and digested under vigorous shaking in collagenase $\mathrm{P}$ ( $1.5 \mathrm{mg} /$ pancreas) for $10 \mathrm{~min}$ at $37^{\circ} \mathrm{C}$. Individual islets were handpicked and cultured in RPMI medium supplemented with $10 \%$ fetal calf serum. Half of the islet material was harvested from culture after 10 days and the remaining islets after 14 days. 
The islets were fixed in a mixture of $2 \%$ paraformaldehyde and $0.25 \%$ glutaraldehyde in $0.1 \mathrm{M}$ phosphate buffer, $\mathrm{pH} 7.4$, and thereafter embedded in Unicryl (British BioCell, Cardiff, UK). Ultrathin sections were immunolabeled with the human-specific antibody A133 against IAPP, diluted 1:100. Bound antibodies were visualized with $10 \mathrm{~nm}$ protein A-gold labeled goat anti-rabbit antibodies (British Biocell).

Kinetic Studies of the Effect of mIAPP on hIAPP Amyloid Fibril Formation

Synthetic mIAPP (Chiron, Australia) and hIAPP (Keck Center, Yale University, New Haven, CT, Yale, PA, USA) were dissolved $10 \mathrm{mg} / \mathrm{ml}$ in dimethylsulfoxide (DMSO). The assay was performed in $50 \mathrm{mM}$ Glycine $-25 \mathrm{mM}$ sodium phosphate buffer, pH 7.0, containing $10 \mu \mathrm{M}$ Thioflavin $\mathrm{T}$, at $20^{\circ} \mathrm{C}(26)$. The final concentration of hIAPP was $1 \mu \mathrm{mol} / 100 \mu \mathrm{l}$ in $4 \%$ DMSO. The fluorescence was measured every $15 \mathrm{~min}$ in an Ascent fluorescence plate reader (Labsystems, Helsinki, Finland) with excitation $444 \mathrm{~nm}$ and emission $485 \mathrm{~nm}$.

\section{Results}

Animals carrying the hIAPP gene showed no major abnormalities. They developed normally and were normoglycemic in the fasting state (e.g., $3.4 \pm 0.2$ and $3.7 \pm 0.6$ in $6 \mathrm{hIAPP}+/+/$ mIAPP- $/$ - and 6 mIAPP- $/$ - 12- to 16-weekold males, respectively). The fasting IAPP plasma concentrations in six male and six

\begin{tabular}{|c|c|c|}
\hline & $\begin{array}{c}\text { hIAPP }+/+ \\
\text { mIAPP }-/- \text { mice }\end{array}$ & $\begin{array}{l}\text { mIAPP-/- } \\
\text { mice }\end{array}$ \\
\hline $\begin{array}{l}\text { Male mice } \\
(n=5)\end{array}$ & $324 \pm 16$ & $255 \pm 33$ \\
\hline $\begin{array}{l}\text { Female mice } \\
(n=5)\end{array}$ & $275 \pm 42$ & $347 \pm 56$ \\
\hline
\end{tabular}

Values are in arbitrary units.

female hIAPP $+/+/$ mIAPP $-/-$ mice, 12-16 weeks old, were $17.8 \pm 3.2$ and $16.6 \pm$ $1.8 \mathrm{pmol} / \mathrm{l}$, respectively. Because IAPP was undetectable in plasma obtained from mIAPP $-/-$ mice, these findings show that hIAPP is expressed and secreted in the hIAPP $+/+/$ mIAPP $-/-$ mice. The islet sizes did not differ between hIAPP $+/+/$ mIAPP $-/-$ mice and mIAPP $-/-$ mice (Table 1 ). Furthermore, the areas of cells expressing insulin glucagon, somatostatin and PP were similar in hIAPP $+/+/$ mIAPP $-/-$ mice and mIAPP $-/-$ animals (Table 2 ).

\section{Weight Gain in hIAPP $+/-/$ mIAPP $-/-$ and mIAPP $-/-$ Male Mice on Normal Diet}

At the age of 3 weeks, there was a tendency toward higher body weight in hIAPP $+/-$ /

Table 2. Compositional analysis of pancreatic islets in hIAPP $+/+$ mIAPP $-/-$ mice and mIAPP $-/-$ mice

\begin{tabular}{|c|c|c|c|c|}
\hline & Insulin & Glucagon & Somatostatin & $\begin{array}{l}\text { Pancreatic } \\
\text { Polypeptide }\end{array}$ \\
\hline $\begin{array}{l}\text { hIAPP }+/+ \text { mIAPP }-I-\text { mice } \\
(n=5) \text { male }\end{array}$ & $67.2 \pm 1.0$ & $7.2 \pm 0.3$ & $4.9 \pm 0.3$ & $4.4 \pm 0.9$ \\
\hline $\begin{array}{l}\text { mIAPP }-/- \text { mice } \\
(n=5) \text { male }\end{array}$ & $65.6 \pm 0.9$ & $8.4 \pm 0.6$ & $6.4 \pm 0.7$ & $4.8 \pm 1.0$ \\
\hline $\begin{array}{l}\text { hIAPP }+/+ \text { mIAPP }-I-\text { mice } \\
(n=5) \text { female }\end{array}$ & $66.4 \pm 1.2$ & $7.6 \pm 0.5$ & $5.8 \pm 0.4$ & $3.2 \pm 0.9$ \\
\hline $\begin{array}{l}\text { mIAPP }-I-\text { mice } \\
(n=5) \text { female }\end{array}$ & $67.3 \pm 0.9$ & $7.8 \pm 0.7$ & $5.2 \pm 0.8$ & $3.6 \pm 0.9$ \\
\hline
\end{tabular}

Values are \% of islet area. 
mIAPP $-/-$ male mice, but this difference did not reach statistical significance $(p=0.08)$. From the age of 10 weeks, the weight curves had changed, and there was a tendency for the mIAPP $-/-$ males to gain more weight than the hIAPP $+/-/$ mIAPP $-/-$ males $(p=0.07)$. At 20 and 30 weeks, these latter differences in weight gain were statistically significant $(p<$ .04 and $p<.03$, respectively).

Weight Gain, Blood Glucose and Islet Amyloid in Male and Female hIAPP +/+/mIAPP - /- on Fat-rich Diet

Both male and female mice on a fat diet gained more weight compared to animals on a normal diet (Fig. 1A). The blood glucose concentrations were significantly higher in both male and female animals on a high-fat diet, in the females from the sixth month and in the males already from the third month (Fig. 1B).

In previous studies, we found that hIAPP transgenic mice on the wild-type mIAPP background given an ordinary diet did not develop islet amyloid at any age, not even when crossed into an ob/ob mouse strain or made insulin-resistant by treatment with corticosteroid (17). However, when mice of the same strain were fed a fat-rich diet, we found that some islet amyloid deposits appeared after the age of 16 months, but only in male mice (unpublished results). In the present study, 19 male and 12 female hIAPP $+/+/$ mIAPP $-/-$ mice were given the same fat-rich diet, and the animals were then killed at different ages and studied for the presence of islet amyloid. No amyloid could be detected at the light microscopic level in the pancreata of the animals sacrificed after 6 months. At the electron microscopic level, immunolabeling for IAPP appeared extracellularly in close vicinity to the betacells in all studied male mice. No typical amyloid deposits were seen, however, at 6 months. In male animals killed after 9 months, amyloid deposits in close contact with the betacells were found at the elec-
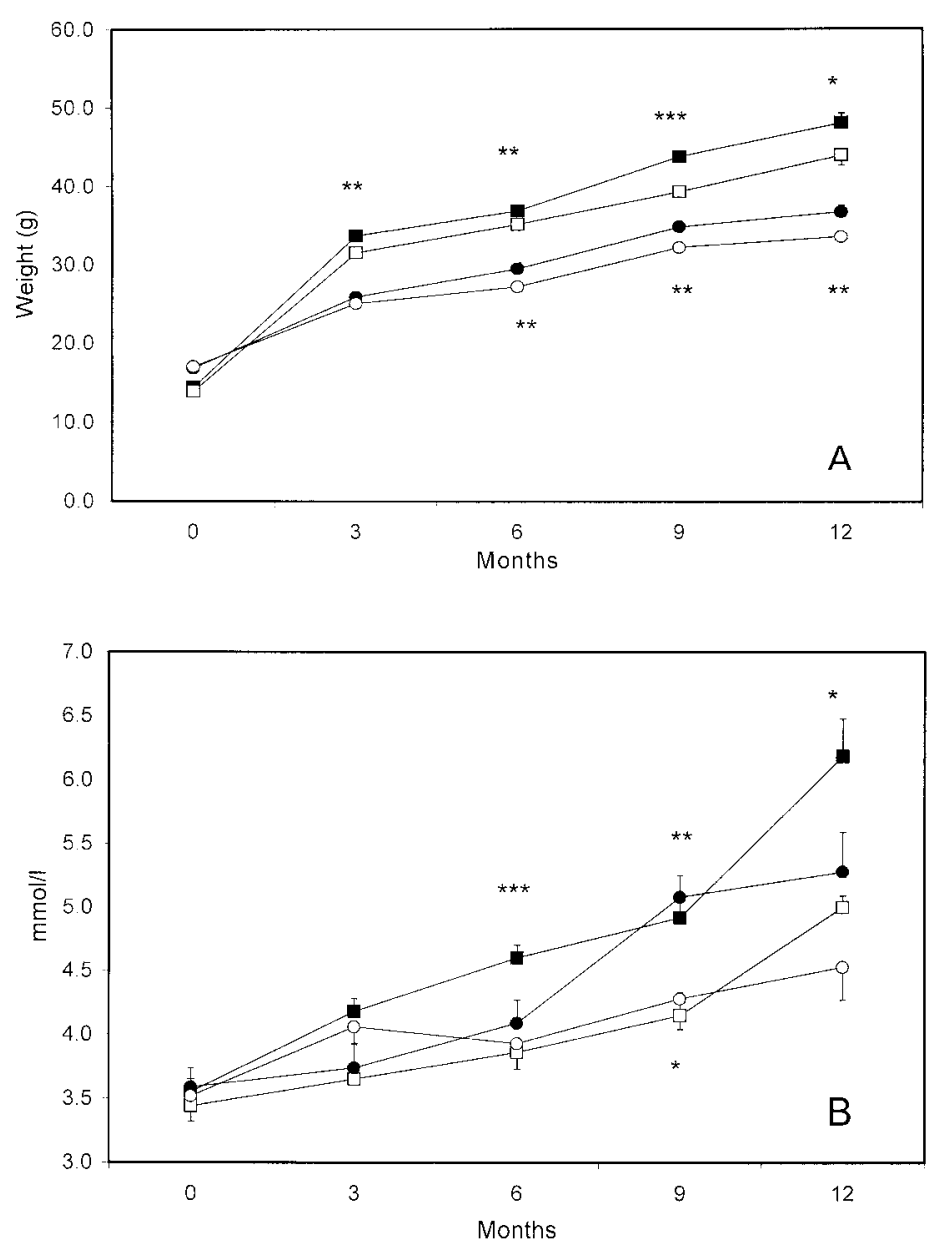

Fig. 1. (A) Weight gain and (B) blood glucose levels determined over a 52-week period in $\square$ hIAPP $+/+$ mIAPP $-/-$ males fed high-fat chow, $\square$ hIAPP+/+mIAPP-/males fed standard chow, hIAPP $+/+$ mIAPP $-/-$ females fed high-fat chow, and $\bigcirc$ hIAPP +/+mIAPP $-/$-females fed standard chow. Values are given as mean \pm SEM. When absent, the error bars lie within symbols. Significance between males above and between females below. ${ }^{*}=p<0.05$; ${ }^{* *}=p<0.01 ;{ }^{* * *}=p<0.001$. 


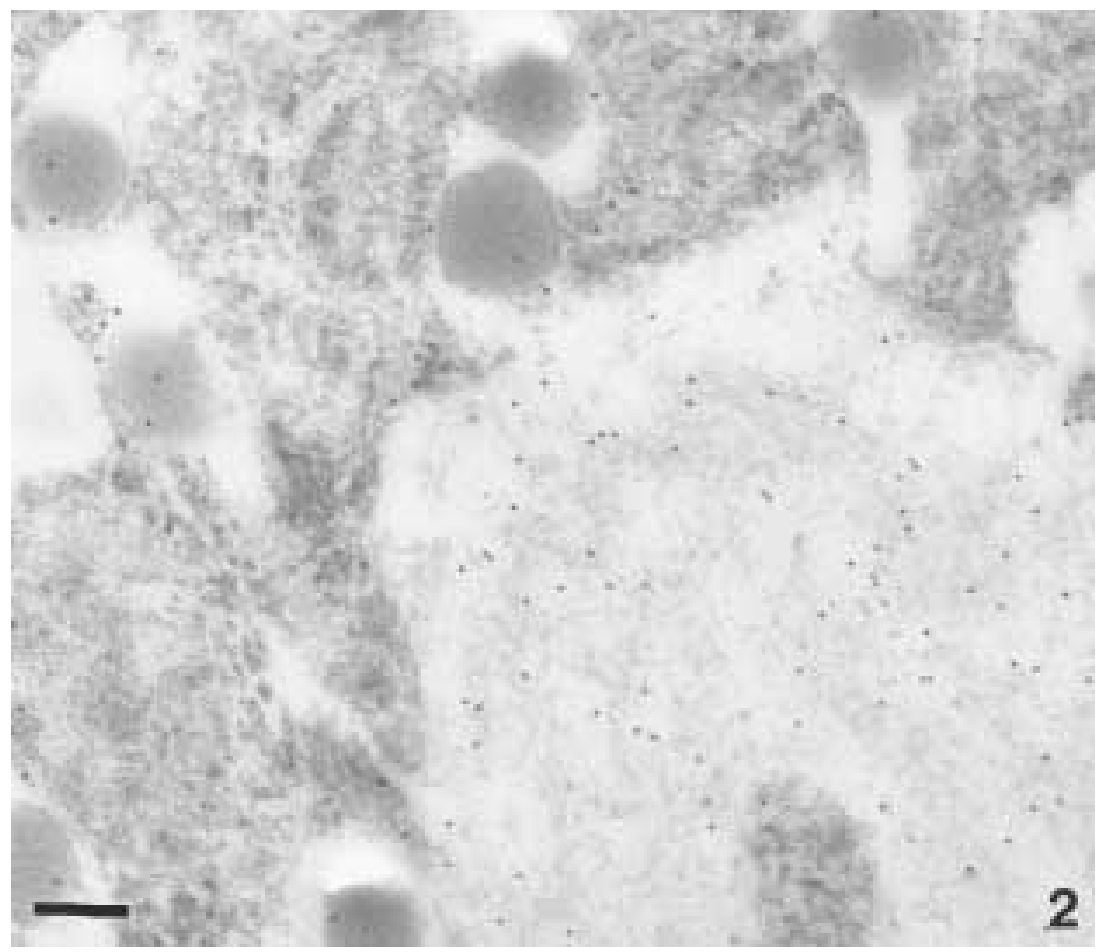

Fig. 2. Islet amyloid in a male mouse fed high-fat chow, labeled with antibodies islet amyloid polypeptide $(10 \mathrm{~nm}$ gold particles). The amyloid is localized extracellularly in close contact to the beta cells. Bar = $200 \mathrm{~nm}$. tron microscopic level in five out of six animals (Fig. 2). After 12 months, three of six studied male mice had large amounts of amyloid in several islets (Fig. 3A). This amyloid stained intensely with Congo red and exhibited green birefringence in polarized light. In some islets, amyloid apparently filled single cells (Fig. 3B). In pancreata from female mice,

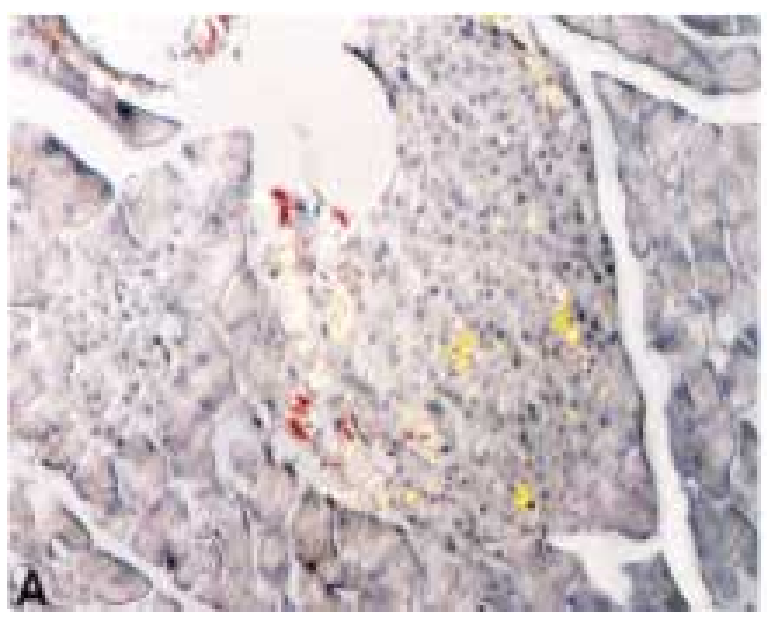

Fig. 3. (A) A pancreatic islet of a hIAPP $+/+$ mIAPP-I-male mouse, fed a high-fat diet for 9 months. Many scattered amyloid deposits are seen within the islet (yellow-red). Congo red in polarized light, examined with partially crossed polars. $200 \times$ magnification. (B) Detail of a no amyloid was detected at any time by light microscopy. However, when pancreatic specimens were studied by electron microscopy, immunolabeling with antibodies specific for IAPP could be seen along the basement membranes in close proximity to the betacells in all animals studied after 9 months of age (Fig. 4).

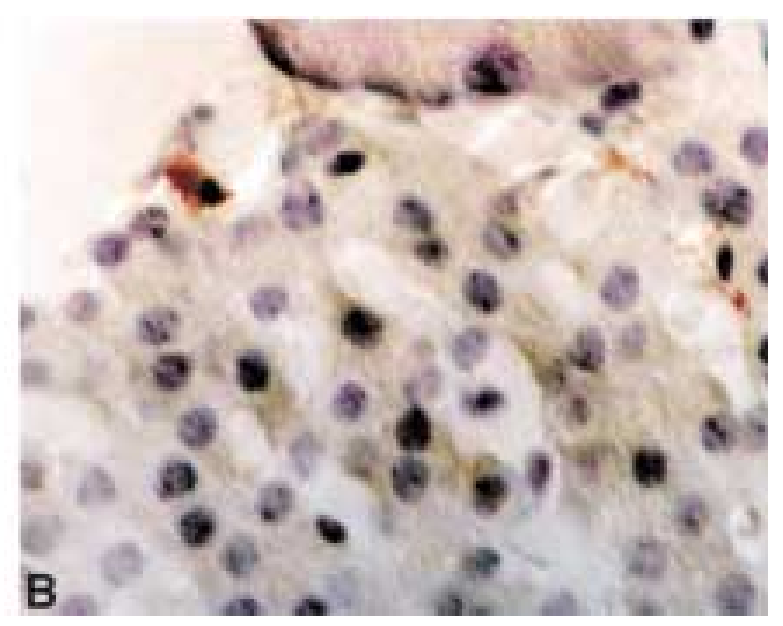

pancreatic islet of the same animal as in (A). Small extracellular amyloid deposits are evident. In addition, a cell, obviously filled with amyloid is seen. Congo red in polarized light, examined with partially crossed polars. $1000 \times$ magnification. 


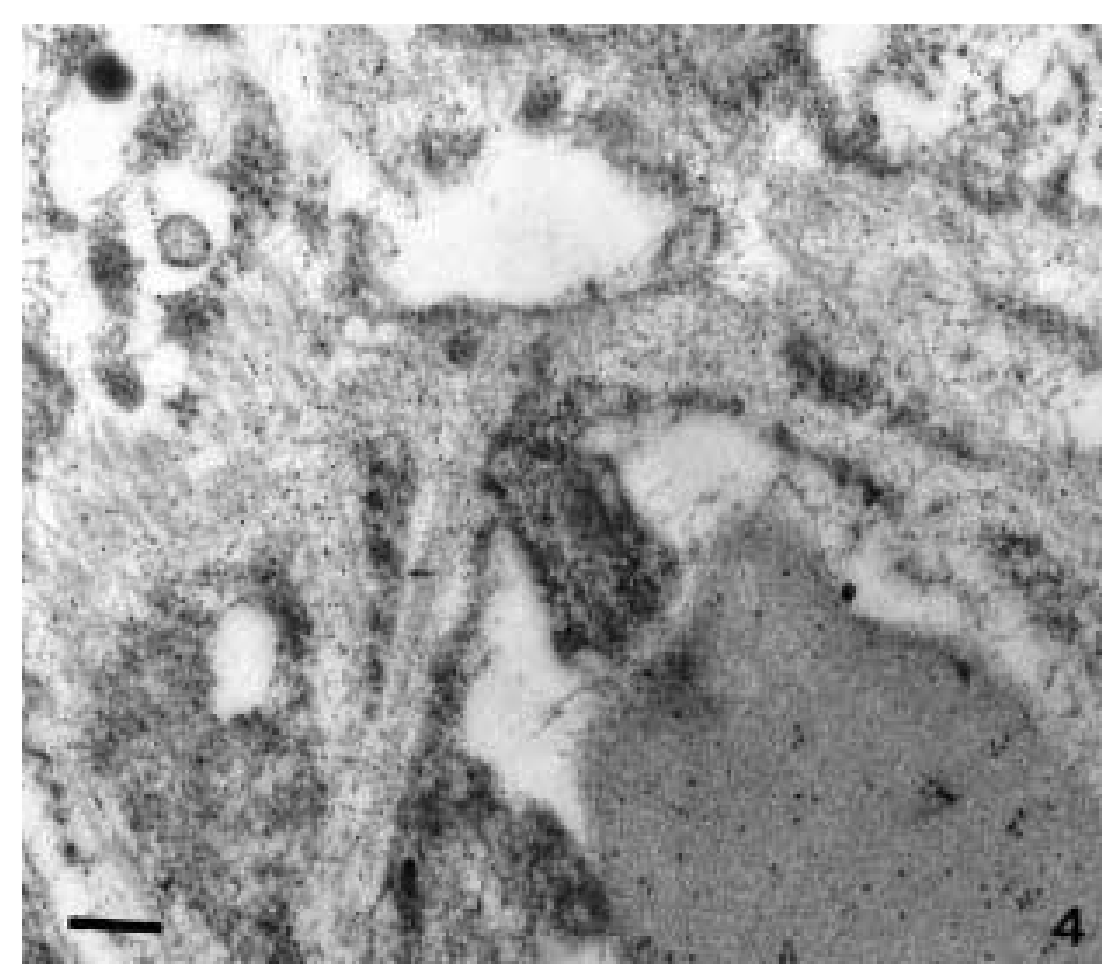

Fig. 4. Part of pancreatic islet of a hIAPP $+/+$ mIAPP $-/-$ female mouse, fed a high-fat diet for 9 months. Part of a beta cell is seen in the upper right corner and a capillary in the lower left corner. Islet amyloid polypeptide immunoreactivity (10 nm gold particles) is seen along the basement membrane but no clear-cut amyloid fibrils occur. Bar $=500 \mathrm{~nm}$.

\section{In Vitro Studies}

Islets from hIAPP $+/-/$ mIAPP $-/-$ mice, cultivated in vitro at a glucose concentration of $11 \mathrm{mM} / \mathrm{l}$ and harvested after 10 and 14 days, contained small extracellular deposits of IAPPimmunoreactive amyloid fibrils. There was no difference in amyloid content between islets from male and from female mice. Most deposits were extracellular but, as found with the in vivo-formed amyloid, minor deposits occurred intracellularly. The extracellular deposits were usually associated with basement membranes and were in close contact with the islet beta cells (data not shown).

\section{Effects of mIAPP on hIAPP Amyloid Fibril Formation}

The effects of the possible interaction of mouse IAPP in fibril formation by human IAPP was tested in an established in vitro fibrillogenesis assay. The result of the fluorescence assay is shown in Figure 5. As shown earlier, mIAPP does not form any fibrils whereas hIAPP spon-

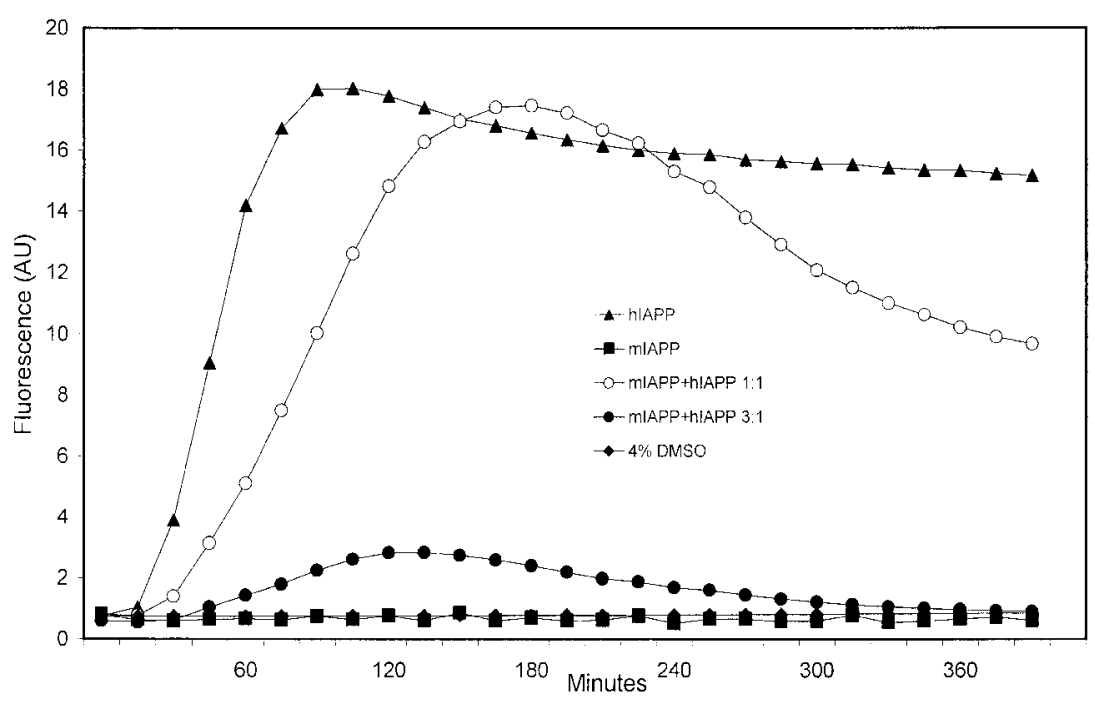

Fig. 5. Fibril formation of human islet amyloid polypeptide (IAPP) assayed by thioflavin T spectroscopy. Human IAPP spontaneously forms amyloid like fibrils whereas mouse IAPP does not. Mouse IAPP inhibits fibril formation of human IAPP in a dose-dependent manner. 
taneously converts into amyloid-like fibrils in vitro. The addition of equal amounts of mIAPP to hIAPP resulted in a significantly prolonged lag phase for fibril formation. When the ratio of mIAPP to hIAPP was further increased, the fibril formation was abolished.

\section{Discussion}

Deposition of amyloid is a highly characteristic pancreatic islet abnormality in type 2 diabetes. Although the biochemical nature of the amyloid has been elucidated and some mechanisms important for fibril formation from IAPP in vitro are known, its formation in vivo is poorly understood. Overexpression of IAPP has been believed to be the sole major factor, but the absence of islet amyloid in several transgenic mouse strains with very high production of hIAPP have contradicted this supposition. Obviously, an amyloidogenic IAPP is a prerequisite for amyloid formation, but additional factors must operate in the IAPP amyloidogenesis. Such factors may be the existence of components facilitating fibril formation (27), but the possible loss of a protective mechanism that normally suppresses conformational changes or aggregation should also be considered. Among the former are heparan sulfate proteoglycan (28) and nidus formation (29), and among the latter, are beta cell granule components (20). A delicate balance among the various beta cell granule components could be important for a nonaggregated state of hIAPP (and any other putative amyloidogenic IAPP species).

We postulated that the presence of mIAPP in beta cell granules of hIAPP transgenic mice might partially inhibit islet amyloid formtion. This supposition was verified in the in vitro experiment where it was found that murine IAPP in ratios of $3 / 1$ or above almost completely abolishes fibrillogenesis. At lower mIAPP concentrations, fibril formation by hIAPP did occur, but it was significantly delayed. We therefore reasoned that a mouse strain expressing hIAPP but lacking mIAPP should more closely mimic the situation in human islets. The finding that islet amyloid occurred earlier than we previously have recorded in this hIAPP transgenic mouse strain clearly supports the theory that the presence of mIAPP affects the formation of amyloid fibrils from hIAPP.
It has been found that amyloid deposits form much faster in islets from hIAPP transgenic mice that had been isolated and cultivated in vitro $(17,18)$ compared to islets in vivo. Also, in normal human isolated islets, transplanted under the renal capsule in nude mice, development of amyloid is rapid, although initially this amyloid occur intracellularly (30). The same rapid development of islet amyloid was recorded in the present study with the hIAPP $+/+/$ mIAPP $-/-$ mice. The reason for the discrepancy between in vivo and in vitro situations is not clear, but it has been suggested that clearance of IAPP may be lower in in vitro-cultivated islets, creating an abnormally high concentration of hIAPP outside beta cells (31). It is also possible that other changes in the extracellular micromilieu of the beta cells occur in vitro that may promote fibril formation.

It has been seen repeatedly that male hIAPP transgenic mice are much more prone to develop islet amyloid as compared to their female counterparts. Whether or not this is a hormonal effect has not been shown. From our in vitro study, however, it is evident that islet amyloid forms to the same degree in islets obtained from either female or male hIAPP transgenic animals.

The slight but statistically significant lower weight gain in male hIAPP $+/-/$ mIAPP $-/-$ mice is of potential interest. Infusion of IAPP has been shown to alter eating habits in rats and to reduce their weight gain, probably by affecting the central nervous system $(32,33)$. However, the mechanism behind the lower weight gain in the mice expressing human IAPP may be multifactorial, and further studies are needed to make clear a role for IAPP.

\section{Acknowledgments}

This work was supported by the Swedish Medical Research Council (project No 5941), the Research Fund of the Swedish Diabetes Association, and the Novo Nordisk Foundation.

\section{References}

1. Westermark P, Johnson KH, O'Brien TD, Betsholtz C. (1992) Islet amyloid polypeptide a novel controversy in diabetes research. Diabetologia 35: 297-303.

2. Schmitz O, Nyholm B, Juhl CB, Lund S, Ørskov L. (1999) Aspects on secretion and actions of 
amylin: interplay between amylin and other hormones. J. Endocrin. Invest. 22(suppl 5): 33-36.

3. Westermark P, Wernstedt C, Wilander E, Sletten K. (1986) A novel peptide in the calcitonin gene related peptide family as an amyloid fibril protein in the endocrine pancreas. Biochem. Biophys. Res. Commun. 140: 827-831.

4. Westermark P. (1996) Islet pathology of noninsulin-dependent diabetes mellitus (NIDDM). Diabet. Med. 13: S46-48.

5. Kahn SE, Andrikopoulos S, Verchere CB. (1999) Islet amyloid: a long-recognized but underappreciated pathological feature of type 2 diabetes. Diabetes 48: 241-253.

6. Janson J, Ashley RH, Harrison D, McIntyre S, Butler PC. (1999) The mechanism of islet amyloid polypeptide toxicity is membrane disruption

by intermediate-sized toxic amyloid particles. Diabetes 48: 491-498.

7. Betsholtz C, Svensson V, Rorsman F, et al. (1989) Islet amyloid polypeptide (IAPP): cDNA cloning and identification of an amyloidogenic region associated with the species-specific occurrence of age-related diabetes mellitus. Exp. Cell Res. 183: 484-493.

8. Westermark P, Engström U, Johnson KH, Westermark GT, Betsholtz C. (1990) Islet amyloid polypeptide: pinpointing amino acid residues linked to amyloid fibril formation. Proc. Natl. Acad. Sci. U.S.A. 87: 5036-5040.

9. D'Alessio DA, Verchere CB, Kahn SE, et al. (1994) Pancreatic expression and secretion of human islet amyloid polypeptide in a transgenic mouse. Diabetes 43: 1457-1461.

10. de Koning EJ, Höppener JW, Oosterwijk C, et al. (1993) Localisation of islet amyloid polypeptide (IAPP) in pancreatic islets of transgenic mice expressing the human or rat IAPP gene. Biochem. Soc. Trans. 21: 26S.

11. Fox N, Schrementi J, Nishi M, et al. (1993) Human islet amyloid polypeptide transgenic mice as a model of non-insulin-dependent diabetes mellitus (NIDDM). FEBS Lett. 323: 4044.

12. Yagui K, Yamaguchi T, Kanatsuka A, et al. (1995) Formation of islet amyloid fibrils in betasecretory granules of transgenic mice expressing human islet amyloid polypeptide/amylin. Eur. J. Endocrinol. 132: 487-496.

13. Verchere CB, D'Alessio DA, Palmiter RD, et al. (1996) Islet amyloid formation associated with hyperglycemia in transgenic mice with pancreatic beta cell expression of human islet amyloid polypeptide. Proc. Natl. Acad. Sci. U.S.A. 93: 34923496.

14. Soeller WC, Janson J, Hart SE, et al. (1998) Islet amyloid-associated diabetes in obese $\mathrm{A}(\mathrm{vy}) / \mathrm{a}$ mice expressing human islet amyloid polypeptide. Diabetes 47: 743-750.
15. Janson J, Soeller WC, Roche PC, et al. (1999) Spontaneous diabetes mellitus in transgenic mice expressing human islet amyloid polypeptide. Proc. Natl. Acad. Sci. U.S.A. 93: 7283-7288.

16. Höppener JWM, Oosterwijk C, Nieuwenhuis MG, et al. (1999) Extensive islet amyloid formation is induced by development of type 2 diabetes mellitus and contributes to its progression: pathogenesis of diabetes in a mouse model. Diabetologia 42: 427-434.

17. Westermark G, Arora MB, Fox N, et al. (1995) Amyloid formation in response to beta cell stress occurs in vitro, but not in vivo, in islets of transgenic mice expressing human islet amyloid polypeptide. Mol. Med. 1: 542-553.

18. de Koning EJ, Morris ER, Hofhuis FM, et al. (1994) Intra- and extracellular amyloid fibrils are formed in cultured pancreatic islets of transgenic mice expressing human islet amyloid polypeptide. Proc. Natl. Acad. Sci. U.S.A. 91: 84678471.

19. MacArthur DLA, de Koning EJP, Verbeek JS, Morris JF, Clark A. (1999) Amyloid fibril formation is progressive and correlates with betacell secretion in transgenic mouse isolated islets. Diabetologia 42: 1219-1227.

20. Westermark P, Li ZC, Westermark GT, Leckström A, Steiner DF. (1996) Effects of beta cell granule components on human islet amyloid polypeptide fibril formation. FEBS. Lett. 379: 203-206.

21. Janciauskiene S, Eriksson S, Carlemalm E, Ahrén B. (1997) B cell granule peptides affect human islet amyloid polypeptide (IAPP) fibril formation in vitro. Biochem. Biophys. Res. Commun. 236: $580-585$.

22. Gebre-Medhin S, Mulder H, Pekny M, et al. (1998) Increased insulin secretion and glucose tolerance in mice lacking islet amyloid polypeptide (amylin). Biochem. Biophys. Res. Commun. 250: 271-277.

23. Westermark GT, Leckström A, Ma Z, Westermark P. (1998) Increased release of IAPP in response to long-term high fat intake in mice. Horm. Metab. Res. 30: 256-258.

24. Westermark GT, Johnson KH, Westermark P. (1999) Staining methods for identification of amyloid in tissue. Meth. Enzymol. 309: 3-25.

25. Ma Z, Westermark GT, Johnson KH, O'Brien TD, Westermark P. (1998) Quantitative immunohistochemical analysis of islet amyloid polypeptide (IAPP) in normal, impaired glucose tolerant, and diabetic cats. Amyloid 5: 255261.

26. LeVine H III. (1999) Quantification of $\beta$-sheet amyloid fibrils structures with thioflavin $\mathrm{T}$. Meth. Enzymol. 309: 274-284.

27. Wisniewski T, Frangione B. (1992) Apolipoprotein $\mathrm{E}$ a pathological chaperone protein in patients with cerebral and systemic amyloid. Neurosci. Lett. 135: 235-238. 
28. Young ID, Ailles L, Narindrasorasak S, Tan R, Kisilevsky R. (1992) Localization of the basement membrane heparan sulfate proteoglycan in islet amyloid deposits in type II diabetes mellitus. Arch. Pathol. Lab. Med. 116: 951-954.

29. Ashburn TT, Lansbury PT. (1993) Interspecies sequence variation affects the kinetics and the thermodynamics of amyloid formation: peptide models of pancreatic amyloid. J. Am. Chem. Soc. 115: 11012-11013.

30. Westermark P, Eizirik D, Pipeleers DG, Hellerström C, Andersson A. (1995) Rapid deposition of amyloid in human islets transplanted into nude mice. Diabetologia 38: 543-549.

31. Clark A, Charge SB, Badman MK, de Koning EJ. (1996) Islet amyloid in type 2 (non-insulindependent) diabetes. A.P.M.I.S. 104: 12-18.

32. Arnelo U, Permert J, Adrian TE, Larsson J, Westermark P, Reidelberger RD. (1996) Chronic infusion of islet amyloid polypeptide causes anorexia in rats. Am. J. Physiol. 271: R 1654-1659.

33. Arnelo U, Blevins JE, Larsson J, et al. (1996) Effects of acute and chronic infusion of islet amyloid polypeptide on food intake in rats. Scand. J. Gastroenterol. 31: 83-89. 